\title{
Prediction of Axillary Nodal Status according to the Axillary Lymph Node to Primary Breast Tumor Maximum Standardized Uptake Value Ratio on ${ }^{18} \mathrm{~F}$-fluorodeoxyglucose Positron Emission Tomography/ Computed Tomography
}

\author{
Woo Young Sun, Young Jin Choi ${ }^{1}$, Young-Jin Song ${ }^{1}$ \\ Department of Surgery, Daejeon St. Mary's Hospital, The Catholic University of Korea College of Medicine, Seoul; ${ }^{1}$ Department of Surgery, Chungbuk \\ National University College of Medicine and Medical Research Institute, Cheongju, Korea
}

Purpose: This study aimed to evaluate the usefulness of the axillary lymph node to primary breast tumor maximum standardized uptake value (SUVmax) ratio in ${ }^{18} \mathrm{~F}$-fluorodeoxyglucose (FDG) positron emission tomography/computed tomography (PET/CT) for predicting the presence of metastasis in axillary lymph nodes. Methods: Herein, 196 consecutive patients with breast cancer who underwent PET/CT before surgery from January 2009 to January 2013 were included. We calculated the axillary lymph node to primary breast tumor SUVmax ratio using PET/CT. The sensitivity, specificity, positive predictive value (PPV), and negative predictive value (NPV) of the ratio were compared with those of nodal SUVmax and axillary ultrasonography. Axillary node metastasis was confirmed with sentinel node biopsy or axillary dissection. Results: The SUVmax ratio (cutoff, 0.14; area under the curve, 0.741) had $55.4 \%$ sensitivity, $91.6 \%$ specificity, $76.6 \%$ PPV, and $80.5 \%$ NPV for predicting axillary node metastasis. No significant difference was observed in terms of predicting axillary node metastasis among the SUVmax ratio, nodal SUVmax, and ultrasonography. In the multivariate analysis, primary tumor size $(p=0.014)$, SUVmax of the primary tumor $(p=0.011)$, axillary ultrasonography findings, nodal SUVmax, and SUVmax ratio were significantly associated with lymph node metastasis $(p<0.001)$. Conclusion: The axillary lymph node to primary breast tumor SUVmax ratio predicted axillary lymph node metastasis, although no significant difference in diagnostic performance was observed between PET/CT and ultrasonography. The axillary lymph node to primary breast tumor SUVmax ratio may be considered an additional method for the preoperative evaluation of axillary lymph node status.

Key Words: Breast neoplasms, Lymph nodes, Neoplasm metastasis, Positron-emission tomography, Ultrasonography

\section{INTRODUCTION}

Breast cancer is the most frequently diagnosed solid malignancy and the second leading cause of cancer-related death in women in Western countries [1]. Among several prognostic factors for breast cancer, lymph node metastasis is recognized as the most important factor associated with survival [2]. Although lymph node metastasis has prognostic value, the precise preoperative evaluation of metastasis is difficult. Although many prognostic factors predicting axillary lymph node metastasis are available [2], a more precise method for predicting metastasis would be useful.

Traditionally, axillary lymph node dissection (ALND) has been the

Correspondence: Young-Jin Song

Department of Surgery, Chungbuk National University College of Medicine and Medical Research Institute, 1 Chungdae-ro, Seowon-gu, Cheongju 28644, Korea Tel: +82-43-269-6361, Fax: +82-43-266-6037, E-mail: yjsong@chungbuk.ac.kr

Received: Aug 11, 2016 Revised: Sep 22, 2016 Accepted: Oct 18, 2016 standard method for axillary nodal assessment; however, this procedure can cause numbness, limitation of motion, and lymphedema [3]. Therefore, sentinel lymph node biopsy (SNB) is widely accepted as a less invasive alternative to ALND for small primary tumors [4].

Positron emission tomography/computed tomography (PET/CT) is a widely used diagnostic tool in several types of malignancies. Because of the enhanced diagnostic performance of PET/CT imaging compared to conventional imaging methods (such as ultrasound, bone scan, and CT alone), it has been used in a variety of settings, including as an approach to staging high-risk patients with early breast cancer [5]. In a recent study, PET/CT was found to be superior to conventional imaging for the detection of distant metastases in patients with untreated stage II or III breast cancer [5]. However, the use of PET/CT as a single imaging procedure is not considered in the routine staging of axillary lymph nodes, as its sensitivity for detecting axillary metastases is too low, ranging from $37 \%$ to $85 \%$ [6,7]. PET/CT does not have suffi- 
cient sensitivity to replace SNB in the detection of axillary lymph node metastasis, but the high specificity of PET/CT is useful when lymph node metastasis is suspected [7,8]. A full diagnostic PET/CT scan has good overall accuracy and high specificity with the potential to triage patients for SNB versus ALND [7].

For the preoperative prediction of axillary lymph node status, visual assessment and semiquantitative analysis with maximum standard uptake value (SUVmax) cutoffs are used $[9,10]$. However, no consistent data have been reported regarding the differentiation of benign from malignant lymph nodes [10]. SUV measurement can differ according to hospital protocols and can be influenced by factors including body composition, blood glucose level, length of the uptake period, and the partial volume effect. SUVmax in lymph nodes is related to the ${ }^{18} \mathrm{~F}$-fluorodeoxyglucose (FDG) avidity of the primary tumor $[11,12]$. Therefore, the axillary lymph node to primary breast tumor SUVmax ratio might predict lymph node status more objectively than nodal SUVmax alone.

In this retrospective study, we assessed the predictive value of the SUVmax ratio in patients with primary breast carcinoma to evaluate whether the SUVmax ratio predicted occult axillary metastasis.

\section{METHODS}

\section{Patients}

From January 2009 to January 2013, 332 patients were diagnosed with breast cancer at Chungbuk National University Hospital, and 239 underwent PET/CT and breast ultrasonography before surgery. Excluded from this group of 239 patients were 26 patients who received neoadjuvant chemotherapy, nine who were diagnosed with bilateral breast cancer, and 28 whose permanent biopsy showed ductal carcinoma in situ. Following these exclusions, study participants included 196 consecutive patients (median age, 51 years; range, 23-80 years) with cT1-3N0-3 stage breast cancer. SUVmax was measured for the primary tumor and axillary lymph nodes. Clinicopathological parameters such as age, estrogen receptor (ER) status, progesterone receptor (PR) status, human epidermal growth factor receptor 2 (HER2) overexpression, Ki-67 overexpression, histological grade, TNM stage, subtype, axillary ultrasonography, primary tumor and nodal SUVmax, and SUVmax ratio were analyzed to determine their usefulness in predicting axillary lymph node metastasis. Relation- ships among SUVmax, SUVmax ratio, ultrasonography, and tumor characteristics were investigated.

Axillary node metastasis was confirmed by SNB ( $\mathrm{n}=131)$ or ALND $(n=65)$. Clinical staging was determined according to the TNM classification of the American Joint Committee on Cancer sixth edition. We classified breast cancer subtypes according to immunohistochemistry and fluorescence in situ hybridization results for ER, PR, and HER2 as follows: luminal A type, ER positive and/or PR positive and HER2 negative; luminal B type, ER positive and/or PR positive and HER2 overexpressed and/or amplified; HER2 type, ER negative and PR negative and HER2 overexpressed and/or amplified; triple-negative breast cancer type, negative for ER, PR, and HER2. Histological types included invasive ductal $(n=174)$, invasive lobular $(\mathrm{n}=11)$, mucinous $(\mathrm{n}=5)$, tubular $(\mathrm{n}=4)$, micropapillary $(\mathrm{n}=1)$, and apocrine carcinoma $(n=1)$. Patients who underwent excisional biopsy prior to surgery were excluded, and none of the study patients received neoadjuvant endocrine therapy. This study was performed in accordance with the guidelines of the local ethics committee.

\section{Axillary ultrasonography}

Ultrasonographic evaluation of axillary lymph nodes was performed by two experienced radiologists with a 10- to $12-\mathrm{MHz}$ linear transducer (Philips Medical Systems, Bothell, USA). If indeterminate, suspicious, or metastatic lymph nodes were detected in the axilla, transverse and longitudinal scans were performed. Longitudinal and transverse diameters and cortical thickness of the nodes were measured. In cases of multiple lymph nodes in the axilla, the most suspicious lymph node was evaluated. Positive findings included lymph nodes that showed: (1) concentric or eccentric cortical thickening of greater than $3 \mathrm{~mm}$; (2) hilar compression and especially absence of the fatty hilum; or (3) length-to-width ratio less than 1.5. Axillary lymph nodes that exhibited any of the three findings were defined as suspicious for axillary lymph node metastasis $[12,13]$.

\section{PET/CT acquisition}

PET/CT (GE Healthcare, Piscataway, USA) scans were performed after conventional breast and axilla imaging. Patients fasted for more than 6 hours before undergoing scanning, and the serum glucose concentration was below $200 \mathrm{mg} / \mathrm{dL}$ prior to ${ }^{18} \mathrm{~F}$-FDG administration. Patients were positioned on a table and a scout scan (topogram) was 

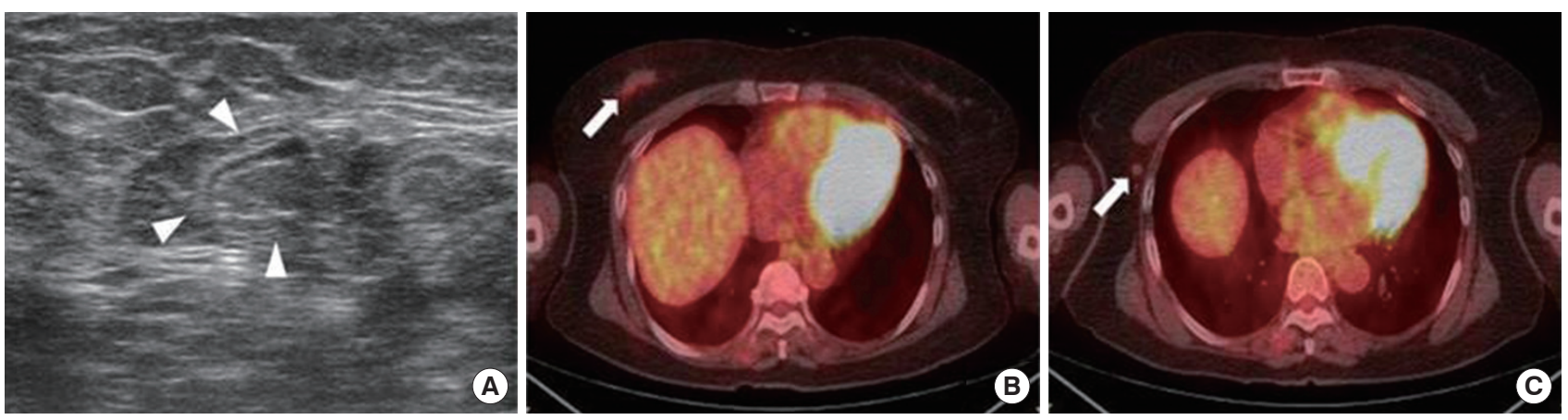

Figure 1. A 66-year-old woman with invasive ductal carcinoma of the right breast. (A) An axillary lymph node (arrowheads) without findings indicative of metastasis such as length-to-width ratio $<1.5$, cortical thickening $>3 \mathrm{~mm}$, or hilar loss is seen by ultrasonography. (B) A hot spot (maximum standard uptake value [SUVmax], 1.3) at the primary breast cancer site (arrow) is shown on positron emission tomography/computed tomography. (C) SUVmax for the right axillary lymph node (arrow) was 1.3 and the SUVmax ratio was 1. Pathological analysis revealed metastasis in the lymph node.

used to define the axial imaging range. Image acquisition began approximately $50 \mathrm{~min}$ after the intravenous injection of $555 \mathrm{MBq}$ of ${ }^{18} \mathrm{~F}-\mathrm{FDG}(7.4 \mathrm{MBq} / \mathrm{kg}, 10 \mathrm{mCi} / \mathrm{kg})$. CT was performed in spiral mode from the base of the skull to the proximal thigh for attenuation correction and image fusion, followed by a three-dimensional caudocranial PET scan. Patients continued shallow breathing during CT acquisition. The emission scan time per bed position was $2.5 \mathrm{~min}$, and six or eight bed positions were employed. CT scans that covered the entire axial imaging range were acquired with a tube voltage of $140 \mathrm{kV}$ and a slice width of $1 \mathrm{~mm}$. CT results were used for PET attenuation correction and fusion. PET results were reconstructed iteratively without and with attenuation correction based on CT results, and reoriented in axial, sagittal, and coronal slices.

\section{PET/CT image analysis}

$\mathrm{PET} / \mathrm{CT}$ images were visually interpreted by an experienced nuclear medicine physician blinded to histopathological, but not clinical information. Lymph nodes were graded as malignant or benign based on functional criteria (increased metabolism relative to surrounding lymph nodes) independent of their size. A lymph node with abnormally increased FDG uptake relative to the surrounding lymph nodes and blood pool activity was considered metastatic regardless of its size. Circulatory regions of interest (ROIs) were manually placed over the entire area of abnormal uptake. Based on the ROI, SUVmax was calculated using the following formula: maximum pixel value within ROI activity $(\mathrm{MBq} / \mathrm{kg}) /($ injected dose $[\mathrm{MBq}] /$ body weight $[\mathrm{kg}])$. The SUVmax ratio was calculated as the proportion of axillary lymph node SUVmax to primary breast tumor SUVmax (Figure 1). The ac- curacy of PET/CT image interpretation was assessed by histopathological analysis (SNB or ALND). We considered the SUVmax in the lymph node to derive from the metastatic lymph node detected by SNB or ALND.

\section{Statistical methods}

The sensitivity, specificity, positive predictive value (PPV), and negative predictive value (NPV) of ultrasonography, SUVmax, and SUVmax ratio were calculated using SPSS version 12.0 (SPSS Inc., Chicago, USA). To evaluate the relationship between SUVmax ratio and the prognostic factors ER, PR, HER2, Ki-67 overexpression, histological grade, or subtype, statistical analyses were performed using Student t-test. To identify an optimal cutoff for the primary tumor and nodal SUVmax and the SUVmax ratio for predicting lymph node metastasis, receiver operating characteristic (ROC) curve analysis was performed. Comparisons between groups including ultrasonography and PET/CT results were performed using the chi-square test. All reported $p$-values were two-sided. A value of $p<0.05$ was considered to be statistically significant.

\section{RESULTS}

Patient characteristics (age, tumor size, lymph node metastasis, stage, histological grade, subtype, receptor status, and Ki-67) and clinical characteristics (axillary ultrasonography, tumor SUVmax, nodal SUVmax, and SUVmax ratio) are presented in Table 1. In 42 patients (21.4\%), axillary ultrasonography showed findings suspicious of metastasis such as a length-to-width ratio $<1.5$, cortical thickening $>3$ 
Table 1. Clinicopathological and radiological characteristics of patients $(\mathrm{n}=196)$

\begin{tabular}{|c|c|}
\hline Characteristic & No. of patients (\%) \\
\hline Age $(y r)^{*}$ & $53.8 \pm 11.4$ \\
\hline Tumor size $(\mathrm{cm})^{*}$ & $1.86 \pm 1.03$ \\
\hline \multicolumn{2}{|l|}{ Histologic grade ${ }^{\dagger}$} \\
\hline 1 & $16(8.2)$ \\
\hline 2 & $120(61.9)$ \\
\hline 3 & $58(29.9)$ \\
\hline \multicolumn{2}{|l|}{ Histologic type } \\
\hline Ductal & $174(88.8)$ \\
\hline Lobular & $11(5.6)$ \\
\hline Mucinous & $5(2.6)$ \\
\hline Tubular & $4(2.0)$ \\
\hline Micropapillary & $1(0.5)$ \\
\hline Apocrine & $1(0.5)$ \\
\hline \multicolumn{2}{|l|}{ TNM stage $^{\dagger}$} \\
\hline 1 & $89(45.4)$ \\
\hline ॥ & $88(44.9)$ \\
\hline III & $19(9.7)$ \\
\hline \multicolumn{2}{|l|}{ Subtype } \\
\hline Luminal A & $119(60.7)$ \\
\hline Luminal B & $21(10.7)$ \\
\hline HER2 & $23(11.8)$ \\
\hline TNBC & $33(16.8)$ \\
\hline \multicolumn{2}{|l|}{ Axillary US } \\
\hline Negative & $154(78.6)$ \\
\hline Positive & $42(21.4)$ \\
\hline Tumor SUVmax* & $4.78 \pm 3.61$ \\
\hline Nodal SUVmax* & $0.87 \pm 2.11$ \\
\hline SUVmax ratio* & $0.14 \pm 0.32$ \\
\hline
\end{tabular}

HER2 = human epidermal growth factor receptor 2; TNBC = triple-negative breast cancer; US = ultrasonography; SUVmax = maximum standardized uptake value.

${ }^{*}$ Mean $\pm \mathrm{SD} ;{ }^{\dagger}$ Number differences reflect missing data.

mm, or loss of hilum. The mean nodal SUVmax of the 196 patients was $0.87 \pm 2.11$ (range, $0-11.9$ ) and the mean primary tumor SUVmax was $4.78 \pm 3.61$ (range, $0.1-17.9$ ). The mean SUVmax ratio was $0.14 \pm$ 0.32 (range, $0-2.32$ ).

No significant differences were observed among the SUVmax ratio, nodal SUVmax, and ultrasonography in prediction of axillary node metastasis. In multivariate analysis, primary tumor size $(p=0.014)$, SUVmax of primary tumor ( $p=0.011$ ), axillary ultrasonography, SUVmax of lymph node, and lymph node to primary tumor SUVmax ratio were significantly associated with lymph node metastasis $(p<0.001)$ (Table 2).

High primary tumor SUVmax was strongly and significantly related to large tumor size $(p=0.02)$, high tumor grade $(p=0.011)$, and
Table 2. Multivariate analysis of predictive factors for lymph node metastasis

\begin{tabular}{|c|c|c|c|c|}
\hline \multirow{2}{*}{ Characteristic } & \multirow{2}{*}{$\begin{array}{c}\text { No. of patients } \\
\text { (\%) }\end{array}$} & \multicolumn{2}{|c|}{ LN metastasis, №. (\%) } & \multirow{2}{*}{$p$-value } \\
\hline & & Negative & Positive & \\
\hline Total No. & 196 & $131(66.8)$ & $65(33.2)$ & \\
\hline Tstage & & & & 0.014 \\
\hline $\mathrm{T} 1$ & $118(60.2)$ & $89(67.9)$ & $29(44.6)$ & \\
\hline $\mathrm{T} 2$ & $74(37.8)$ & $38(29)$ & $36(55.4)$ & \\
\hline $\mathrm{T} 3$ & $4(2.0)$ & $4(3.1)$ & 0 & \\
\hline ER & & & & 0.712 \\
\hline Negative & $49(25.0)$ & $31(23.7)$ & $18(27.7)$ & \\
\hline Positive & $147(75.0)$ & $100(76.3)$ & $47(72.3)$ & \\
\hline PR & & & & 0.871 \\
\hline Negative & $65(33.2)$ & $43(32.9)$ & $22(33.8)$ & \\
\hline Positive & $131(66.8)$ & $88(67.1)$ & $43(66.2)$ & \\
\hline HER2 & & & & 0.494 \\
\hline Negative & $164(83.7)$ & $108(82.4)$ & $56(86.2)$ & \\
\hline Positive & $32(16.3)$ & $23(17.6)$ & $9(13.8)$ & \\
\hline Ki-67 & & & & 0.77 \\
\hline Low, $<15 \%$ & $134(68.4)$ & $93(71)$ & $41(63.1)$ & \\
\hline High, $\geq 15 \%$ & $62(31.6)$ & $38(29)$ & $24(36.9)$ & \\
\hline Axillary US & & & & $<0.001$ \\
\hline Negative & $154(78.6)$ & $123(93.9)$ & $31(47.7)$ & \\
\hline Positive & $42(21.4)$ & $8(6.1)$ & $34(52.3)$ & \\
\hline Nodal SUVmax & & & & $<0.001$ \\
\hline$<1$ & $150(76.5)$ & $120(91.6)$ & $30(46.2)$ & \\
\hline$\geq 1$ & $46(23.5)$ & $11(8.4)$ & $35(53.8)$ & \\
\hline SUVmax ratio & & & & $<0.001$ \\
\hline$<0.14$ & $148(75.5)$ & $119(90.8)$ & $29(44.6)$ & \\
\hline$\geq 0.14$ & $48(24.5)$ & $12(9.2)$ & $36(55.4)$ & \\
\hline
\end{tabular}

$E R=$ estrogen receptor; $\mathrm{PR}=$ progesterone receptor; $\mathrm{HER} 2=$ human epidermal growth factor receptor 2; US= ultrasonography; SUVmax= maximum standardized uptake value.

Table 3. Cutoff value, sensitivity, specificity, and AUC area of US, nodal SUVmax, and SUVmax ratio to predict nodal status

\begin{tabular}{lcccc}
\hline & Cutoff & $\begin{array}{c}\text { Sensitivity } \\
(\%)\end{array}$ & $\begin{array}{c}\text { Specificity } \\
(\%)\end{array}$ & $\begin{array}{c}\text { AUC } \\
(95 \% \mathrm{Cl})\end{array}$ \\
\hline Axillary US & $>0$ & 52.3 & 93.9 & $0.731(0.649-0.813)$ \\
Nodal SUVmax & 1 & 53.8 & 93.9 & $0.746(0.664-0.828)$ \\
SUVmax ratio & 0.14 & 55.4 & 91.6 & $0.741(0.659-0.823)$
\end{tabular}

$\mathrm{AUC}=$ area under the curve; US= ultrasonography; SUVmax= maximum standardized uptake value.

nodal SUVmax $(p<0.001)$. Node positivity $(p=0.026)$, and primary tumor SUVmax $(p<0.001)$ were associated with high nodal SUVmax in multivariate analysis. No differences were observed between primary tumor and nodal SUVmax according to hormone receptor status, tumor subtype, HER2 overexpression, or high Ki-67 (data not shown).

For the nodal SUVmax method (cutoff, 1; area under the curve, 

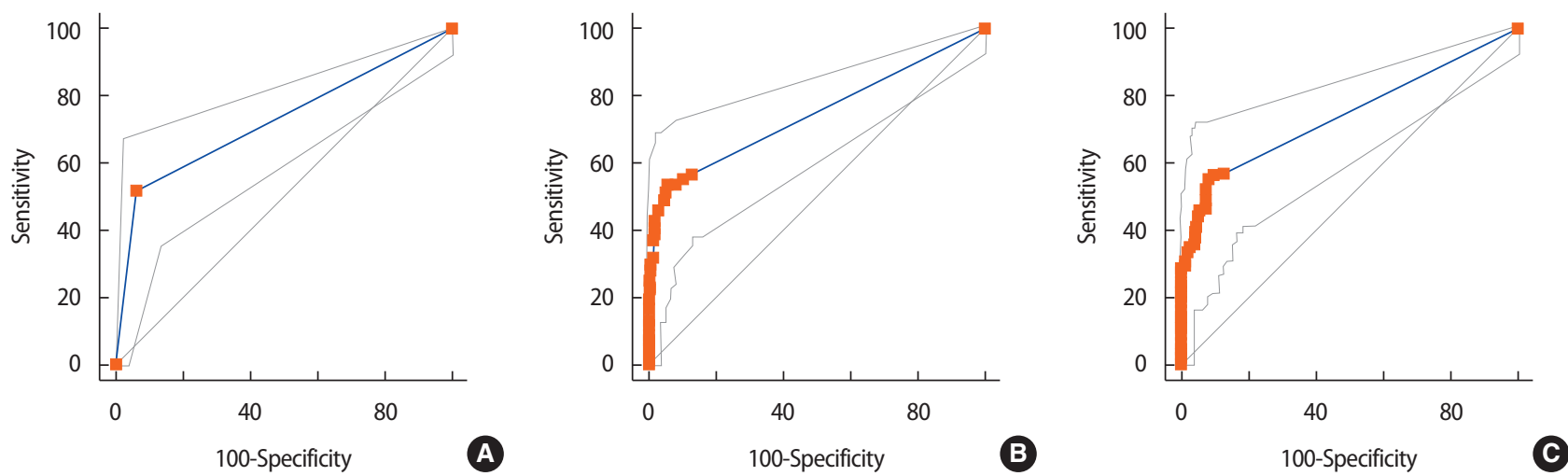

Figure 2. Receiver operating characteristic curve for predicting axillary node metastasis in patients with breast cancer. No significant difference was seen in prediction of lymph node metastasis among axillary ultrasonography (A), lymph node maximum standard uptake value (SUVmax) (B), and SUVmax ratio (C) of axillary lymph node to primary breast tumor on positron emission tomography/computed tomography.

Table 4. Overall sensitivity, specificity, PPV, and NPV of US, SUVmax, and SUVmax ratio to predict nodal status according to $T$ stage of breast cancer

\begin{tabular}{llcccc}
\hline & Tstage & Sensitivity & Specificity & PPV & NPV \\
\hline Axillary US & T1 & 44.8 & 96.6 & 81.3 & 84.3 \\
& T2, T3 & 58.3 & 88.1 & 80.8 & 71.2 \\
\multirow{5}{*}{ Nodal SUVmax } & All & 52.3 & 93.9 & 81.0 & 79.9 \\
& T1 & 44.8 & 98.9 & 92.9 & 84.6 \\
& T2, T3 & 63.9 & 85.7 & 79.3 & 73.5 \\
SUVmax ratio & All & 53.8 & 93.9 & 81.4 & 80.4 \\
& T1 & 48.3 & 92.1 & 66.7 & 84.5 \\
& T2, T3 & 63.9 & 90.5 & 85.2 & 74.5 \\
& All & 55.4 & 91.6 & 76.6 & 80.5 \\
\hline
\end{tabular}

$\mathrm{PPV}=$ positive predictive value; $\mathrm{NPV}=$ negative predictive value; $\mathrm{US}=\mathrm{ultra}-$ sonography; SUVmax = maximum standardized uptake value.

0.746), sensitivity was $53.8 \%$, specificity was $93.9 \%$, PPV was $81.4 \%$, and NPV was $80.4 \%$. ROC curve analysis identified an SUVmax ratio of 0.14 (sensitivity, 55.4\%; specificity, 91.6\%; PPV, 76.6\%; NPV, 80.5\%; area under the curve, 0.741 ) as the optimal cutoff for predicting axillary node metastasis (Table 3, Figure 2). The overall sensitivity of ultrasonography, SUVmax, and SUVmax ratio for predicting nodal status differed for each method according to breast cancer T stage (Table 4).

\section{DISCUSSION}

We evaluated the utility of PET/CT for the detection of axillary lymph node metastases in patients with breast cancer undergoing SNB or ALND. The sensitivity of PET/CT is lower in patients with early breast cancer and clinically negative axillary nodes. In a prospective study, Veronesi et al [6]. compared preoperative PET imaging with
SNB and reported a sensitivity of only 37\% with PET imaging. Gil-Rendo et al. [14] showed a sensitivity of $100 \%$ for detecting lymph node metastasis in patients with grade III malignancy and primary tumor SUVmax higher than 3.5. Straver et al. [15] reported a sensitivity of $97 \%$ with PET/CT for detecting axillary involvement. The sensitivity of PET/CT was 53.8\% in the current study. The SUVmax of small lymph nodes with metastasis might be underestimated because of partial-volume effects and the limited resolution of PET/CT. Higher false negative rates were noted in patients with smaller tumors, which may result from the low SUVmax of metastatic axillary lymph nodes derived from primary breast cancers [16]. Our results also showed that PET/CT was more sensitive for larger tumors (T1 vs. T2, T3).

The low sensitivity and inability to detect micrometastases can be explained by the relatively low spatial resolution of PET. This imaging modality does not allow the detection of micrometastases found using serial sectioning and immunohistochemistry during the pathological assessment of sentinel nodes. Other factors that could influence the sensitivity of PET/CT include intrinsic tumor characteristics such as grade and type $[6,17]$. These results suggest that $\mathrm{SNB}$ is superior to PET/CT in the detection of axillary metastasis in patients with early-stage breast cancer. In the present study, the specificity of PET/ CT was 93.9\%, similar to the findings of other PET/CT studies $[15,18]$. SNB may not be necessary in patients with findings highly suspicious for axillary lymph node metastasis on PET/CT; these patients could undergo complete ALND as the primary procedure, an approach that would reduce time and costs.

Higher SUV correlates with a variety of adverse prognostic vari- 
ables, including high proliferation index, tumor grade, HER2 expression, ER negativity, and greater numbers of circulating tumor cells $[19,20]$. However, no clear relationship was observed between SUVmax and ER status, HER2 overexpression, or Ki-67 in the present study, although a relationship was observed between SUVmax and tumor grade. High primary tumor SUVmax was related to large tumor size and nodal SUVmax, consistent with the findings of previous studies [21,22]. In breast cancer, tumor histopathology indicates that lobular carcinoma is associated with lower FDG uptake than ductal carcinoma [21]. The results of the current study revealed no differences in FDG uptake of the primary cancer or lymph nodes according to histologic type. However, only 11 patients (5.6\%) with invasive lobular carcinoma were included in this study. Studies with larger sample sizes are needed to determine statistical significance.

In a variety of malignancies, higher SUVmax at diagnosis is associated with inferior survival $[23,24]$. In one study, a group of patients with high primary tumor SUVmax had significantly worse prognosis than a group with low primary tumor SUVmax. In another study, high nodal SUVmax showed a significant association with poor prognosis in patients with breast cancer $[25,26]$. Consistent with these reports that primary tumor and nodal SUVmax are significantly associated with prognosis in patients with breast cancer, primary tumor and nodal SUVmax, and the SUVmax ratio, were significantly associated with lymph node metastasis in the present study. Analysis of the relationship between FDG uptake parameters and treatment outcomes and survival was beyond the scope of this study.

We compared ultrasonography with PET/CT in terms of the detection of occult axillary metastasis. No statistical differences were observed between the methods in the current study. Although SUVmax is a suitable method for PET/CT image quantification, it should be considered a semiquantitative method by visual assessment. We experienced some problems with objectivity in the detection and validation of lymph nodes on PET/CT in the present study, because we were unable to validate the assessment of lymph nodes with abnormal uptake as metastatic on the basis of pathological results. We considered SUVmax to indicate the status of the metastatic lymph node examined via SNB or ALND. We did not determine intraobserver or interobserver variability for SUVmax, a limitation inherent to the semiquantitative nature of this study. SUV measurements can be influenced by factors including body composition, blood glucose level, length of uptake period, partial volume, and protocols [10]. The optimal cutoff was 0.14 in the present study. However, no definite cutoff has been established for the SUVmax ratio. Further research is warranted to define specific cutoffs for the SUVmax ratio.

We evaluated the ability of the axillary lymph node to primary breast tumor SUVmax ratio to detect occult metastases. The results were in agreement with the findings of previous studies that demonstrated poor sensitivity of this approach for detecting axillary node metastases in early breast cancer [17,27]. PET/CT did not have sufficient sensitivity to replace SNB in the detection of axillary lymph node metastasis. This study is one of the few conducted with the aim of assessing the predictive value of the SUVmax ratio in patients with breast cancer. The axillary lymph node to primary breast tumor SUVmax ratio might be a more objective method than axillary ultrasonography, which is operator-dependent, for predicting lymph node metastasis.

In conclusion, the present study revealed that the axillary lymph node to primary breast tumor SUVmax ratio could be used to predict lymph node metastasis, although no significant difference in diagnostic performance was observed between PET/CT and ultrasonography. The axillary lymph node to primary breast tumor SUVmax ratio may be considered an additional method for the preoperative evaluation of axillary lymph node status.

\section{CONFLICT OF INTEREST}

The authors declare that they have no competing interests.

\section{REFERENCES}

1. Jemal A, Siegel R, Ward E, Hao Y, Xu J, Murray T, et al. Cancer statistics, 2008. CA Cancer J Clin 2008;58:71-96.

2. Chua B, Ung O, Taylor R, Boyages J. Frequency and predictors of axillary lymph node metastases in invasive breast cancer. ANZ J Surg 2001;71:723-8.

3. Ivens D, Hoe AL, Podd TJ, Hamilton CR, Taylor I, Royle GT. Assessment of morbidity from complete axillary dissection. Br J Cancer 1992;66:136-8.

4. Lyman GH, Giuliano AE, Somerfield MR, Benson AB 3rd, Bodurka DC, Burstein HJ, et al. American Society of Clinical Oncology 
guideline recommendations for sentinel lymph node biopsy in early-stage breast cancer. J Clin Oncol 2005;23:7703-20.

5. Fuster D, Duch J, Paredes P, Velasco M, Muñoz M, Santamaría G, et al. Preoperative staging of large primary breast cancer with $[18 \mathrm{~F}]$ fluorodeoxyglucose positron emission tomography/computed tomography compared with conventional imaging procedures. J Clin Oncol 2008;26:4746-51.

6. Veronesi U, De Cicco C, Galimberti VE, Fernandez JR, Rotmensz N, Viale G, et al. A comparative study on the value of FDG-PET and sentinel node biopsy to identify occult axillary metastases. Ann Oncol 2007;18:473-8.

7. Heusner TA, Kuemmel S, Hahn S, Koeninger A, Otterbach F, Hamami ME, et al. Diagnostic value of full-dose FDG PET/CT for axillary lymph node staging in breast cancer patients. Eur J Nucl Med Mol Imaging 2009;36:1543-50.

8. Quon A, Gambhir SS. FDG-PET and beyond: molecular breast cancer imaging. J Clin Oncol 2005;23:1664-73.

9. Kim J, Lee J, Chang E, Kim S, Suh K, Sul J, et al. Selective sentinel node plus additional non-sentinel node biopsy based on an FDGPET/CT scan in early breast cancer patients: single institutional experience. World J Surg 2009;33:943-9.

10. Carkaci S, Adrada BE, Rohren E, Wei W, Quraishi MA, Mawlawi O, et al. Semiquantitative analysis of maximum standardized uptake values of regional lymph nodes in inflammatory breast cancer: is there a reliable threshold for differentiating benign from malignant? Acad Radiol 2012;19:535-41.

11. Lovrics PJ, Chen V, Coates G, Cornacchi SD, Goldsmith CH, Law C, et al. A prospective evaluation of positron emission tomography scanning, sentinel lymph node biopsy, and standard axillary dissection for axillary staging in patients with early stage breast cancer. Ann Surg Oncol 2004;11:846-53.

12. Ahn JH, Son EJ, Kim JA, Youk JH, Kim EK, Kwak JY, et al. The role of ultrasonography and FDG-PET in axillary lymph node staging of breast cancer. Acta Radiol 2010;51:859-65.

13. Duchesne N, Jaffey J, Florack P, Duchesne S. Redefining ultrasound appearance criteria of positive axillary lymph nodes. Can Assoc Radiol J 2005;56:289-96.

14. Gil-Rendo A, Zornoza G, García-Velloso MJ, Regueira FM, Beorlegui C, Cervera M. Fluorodeoxyglucose positron emission tomography with sentinel lymph node biopsy for evaluation of axillary involve- ment in breast cancer. Br J Surg 2006;93:707-12.

15. Straver ME, Aukema TS, Olmos RA, Rutgers EJ, Gilhuijs KG, Schot ME, et al. Feasibility of FDG PET/CT to monitor the response of axillary lymph node metastases to neoadjuvant chemotherapy in breast cancer patients. Eur J Nucl Med Mol Imaging 2010;37:106976.

16. Soret M, Bacharach SL, Buvat I. Partial-volume effect in PET tumor imaging. J Nucl Med 2007;48:932-45.

17. Barranger E, Grahek D, Antoine M, Montravers F, Talbot JN, Uzan S. Evaluation of fluorodeoxyglucose positron emission tomography in the detection of axillary lymph node metastases in patients with early-stage breast cancer. Ann Surg Oncol 2003;10:622-7.

18. Hodgson NC, Gulenchyn KY. Is there a role for positron emission tomography in breast cancer staging? J Clin Oncol 2008;26:712-20.

19. De Cicco C, Gilardi L, Botteri E, Fracassi SL, Di Dia GA, Botta F, et al. Is $[18 \mathrm{~F}]$ fluorodeoxyglucose uptake by the primary tumor a prognostic factor in breast cancer? Breast 2013;22:39-43.

20. Basu S, Chen W, Tchou J, Mavi A, Cermik T, Czerniecki B, et al. Comparison of triple-negative and estrogen receptor-positive/progesterone receptor-positive/HER2-negative breast carcinoma using quantitative fluorine-18fluorodeoxyglucose/positron emission tomography imaging parameters: a potentially useful method for disease characterization. Cancer 2008;112:995-1000.

21. Sanli Y, Kuyumcu S, Ozkan ZG, Işik G, Karanlik H, Guzelbey B, et al. Increased FDG uptake in breast cancer is associated with prognostic factors. Ann Nucl Med 2012;26:345-50.

22. Hahn S, Hecktor J, Grabellus F, Hartung V, Pöppel T, Kimmig R, et al. Diagnostic accuracy of dual-time-point 18F-FDG PET/CT for the detection of axillary lymph node metastases in breast cancer patients. Acta Radiol 2012;53:518-23.

23. Liao CT, Chang JT, Wang HM, Ng SH, Hsueh C, Lee LY, et al. Preoperative $[18 \mathrm{~F}]$ fluorodeoxyglucose positron emission tomography standardized uptake value of neck lymph nodes predicts neck cancer control and survival rates in patients with oral cavity squamous cell carcinoma and pathologically positive lymph nodes. Int J Radiat Oncol Biol Phys 2009;74:1054-61.

24. Kidd EA, Siegel BA, Dehdashti F, Grigsby PW. Pelvic lymph node F-18 fluorodeoxyglucose uptake as a prognostic biomarker in newly diagnosed patients with locally advanced cervical cancer. Cancer 2010;116:1469-75. 
25. Inoue T, Yutani K, Taguchi T, Tamaki Y, Shiba E, Noguchi S. Preoperative evaluation of prognosis in breast cancer patients by [(18) F]2-Deoxy-2-fluoro-D-glucose-positron emission tomography. J Cancer Res Clin Oncol 2004;130:273-8.

26. Song BI, Lee SW, Jeong SY, Chae YS, Lee WK, Ahn BC, et al. 18F-FDG uptake by metastatic axillary lymph nodes on pretreat- ment PET/CT as a prognostic factor for recurrence in patients with invasive ductal breast cancer. J Nucl Med 2012;53:1337-44.

27. Zornoza G, Garcia-Velloso MJ, Sola J, Regueira FM, Pina L, Beorlegui C. 18F-FDG PET complemented with sentinel lymph node biopsy in the detection of axillary involvement in breast cancer. Eur J Surg Oncol 2004;30:15-9. 